\title{
Artificial Intelligence for Environmental and Climate Protection
}

There are numerous ways in which applications powered by intelligent algorithms can be used to benefit the environment and protect the climate. But to ensure an overall positive impact on the environment, Artificial Intelligence applications should be used with caution, and most importantly, only promoted in areas where they really make sense.

By Sarah-Indra Jungblut

A rtificial Intelligence (AI) has long since found its place in our everyday lifes, in companies and in industry - whether it is as a search engine, a personal voice assistant or robots and autonomous machines programmed for specific activities. However, projects, start-ups, companies, and research projects that are developing and testing the use of AI to protect the environment or the climate are still an exception: A keyword search on Crunchbase by reset.org in June 2020 for the DBUfunded publication Greenbook (1): Künstliche Intelligenz - Können wir mit Rechenleistung unseren Planeten retten? [Artificial Intelligence - Can Computing Power Save Our Planet?] (RESET 2020) revealed around 400 AI start-ups with a sustainability focus - compared to a total of almost 20,000 AI start-ups worldwide. A similar picture emerges in research. Even though there is an increasing number of studies that focuses on individual areas of application of AI in the context of sustainability, so far there are no studies at either a European or an international level that enable us to thoroughly evaluate research activities in this field. A short study commissioned by the German Environment Agency comes to the same conclusion (UBA 2019).

When looking at specific AI applications, it becomes clear that there is a large range of different areas where it is possible to apply AI to protect the environment and the climate, and that intelligent algorithms are in fact already proving to be highly effective (RESET 2020).

\section{Learning algorithms for a smart energy grid}

AI technologies harbour great opportunities for more effective management of the energy market, which is becoming more and more complex because of the increased use of renewable energies. Machine learning can help us, through simulations and forecasts for example, to gain a better understanding of the structure of the energy market and to better align and coordinate electricity production and consumption. AI-based systems can, for example, use data on the availability of storage, demand-side management, and power-to- $\mathrm{X}$ technologies to enable grid operators to use electricity from renewable energy sources instead of curtailing it as "surplus electricity". It is conceivable that this could create a kind of platform that enables transparent communication between all actors in the energy system, from citizens, local actors and municipal suppliers to grid-operating actors and energy supply companies.

The open-source project PowerTAC [1], which has developed a sophisticated AI-based simulation, shows what these approaches could look like in real life - by mimicking the interactions of energy suppliers and consumers, and providing potential avenues for balance and regulation in a system with a fluctuating energy supply. PowerTAC is now the largest opensource smart grid project in the world and the software has been downloaded over 10.000 times. It allows decision-makers and industry actors to gain a better understanding of the mechanisms required to implement and realise a decarbonised, decentralised, and digitalised energy system in the future.

For consumers, and also companies, to be able to recognise at what time electricity is produced most sustainably and to adjust their energy consumption accordingly, the British transmission network National Grid, together with Oxford University, the Environmental Defense Fund Europe and WWF, has developed a kind of "weather report" for clean electricity. The Carbon Intensity Forecast [2] uses regression models based on machine learning to predict the $\mathrm{CO}_{2}$ intensity of electricity. This enables companies, for example, to use environmentally friendly electricity or to charge electric vehicles at times when there is a high proportion of green electricity in the mix.

\section{Enhancing sustainable buildings with Artificial Intelligence}

AI solutions can also provide forecasting, diagnosis, and control systems to help us operate existing buildings in a more energy-efficient and climate-friendly way. For example, AI can be used to link IoT (Internet of Things) data, electricity prices, weather data, data from airborne or terrestrial laser scanning, mobile mapping, RGB-D cameras (depth cameras), image matching or multi-beam echo sounding. The exchange of all 
this information between the relevant components of an energy system makes it possible to adjust energy demand to energy supply. In practice, this means that heat pumps or refrigerators, for instance, start up when there is a lot of cheap electricity from renewable energies available.

Various start-ups and companies have begun to develop solutions in this area. At the heart of the approach adopted by Bractlet [3] of Austin, Texas, is the understanding that every building is a unique "ecosystem" with its own peculiarities and irregularities. Bractlet therefore pools data from different sources, such as utility bills, architectural documents, weather and realtime power consumption data, and uses machine learning algorithms to create a building's "digital twin". This is a simulation model that is almost identical to the actual building in terms of energy consumption and can be used to identify the most efficient energy-saving measures. By providing a space for digital experimentation, the company aims to minimise the risk of energy-saving products being installed incorrectly or not in the optimal location in a building's infrastructure, and ultimately being ineffective. According to the company, the suggested energy-saving measures could reduce energy costs by an average of around $30 \%$.

In principle, the "digital twins" concept can be transferred to almost all kinds of physical objects, geographical regions or infrastructures. It is also possible that these simulation models could be used for geospatial solutions such as environmental monitoring, disaster management or urban planning (Döllner 2020).

Across all of the above examples, the more information is available, the better machine learning works. For example, to optimise the energy efficiency of apartments to fit the habits of the users, many analysis and forecasting tools collect data on how long residents or users spend in which room, when they are at home and what temperature they prefer, as the example of Leanheat [4] shows. An intelligent, self-learning control system combines the needs of the residents of an apartment building with the current weather conditions and the learned thermodynamics of the building.

\section{Data protection and energy consumption}

It is important to protect personal data in applications such as these. Leanheat uses end-to-end encryption for all data. An important approach to maintaining data protection is, of course, to collect as little data as possible from the outset (data avoidance and data economy), and also to ensure data protection-friendly technology design and appropriate default settings (privacy by design and privacy by default) (c. f. Pohl et al. this issue, Frick et al this issue). The GDPR already addresses these aspects to some extent. Data trust models, such as those proposed by the Stiftung Neue Verantwortung, go even further (Blankertz et al. 2020).

Another key aspect that influences the effectiveness of AI-based applications in terms of energy efficiency is their own energy consumption. Deep learning models in particular require large amounts of energy when being trained. It is therefore important to check in advance how much energy is being used in relation to the possible savings (c. f. Rohde et al. this issue).

\section{Efficient use of resources}

As the applications presented show, intelligent algorithms are particularly helpful in areas where a lot of data flows together and can be evaluated. This also makes Industry 4.0 a suitable (playing) field for the technology: In today's industry, production machines and industrial robots work together with planning and control software. Considerable amounts of data are generated across all processes and conventional methods of analysis quickly reach their limits. The intelligent algorithms are able to link huge amounts of a wide variety of data - giving us the opportunity to control machines and processes more efficiently and thus reduce energy consumption, resource expenditure and reject rates in the production process.

When it comes to cross-company value chains, the application of AI goes one step further. While most Industry 4.0 approaches as we know them today stop at company boundaries or direct supplier and customer interfaces, the use of AI can enable the linking of product and production-related data within (or even beyond) an industry's entire value chain. Tracking the entire life cycle of products and resource flows could prove to be an effective tool on the path towards a truly circular economy. The Environmental Digital Agenda from the German Federal Ministry for the Environment (BMU 2020), for instance, proposes the idea of developing a "digital product passport" for more transparency about the environmental impacts of different products. This would use intelligent algorithms to collect and provide information about where the raw materials come from, the working conditions it was produced under, how much $\mathrm{CO}_{2}$ was generated in the process and information about how to recycle it.

Launched in February 2020, the REIF project [5] aims to use Artificial Intelligence to help reduce food losses in the dairy, meat and baked goods sectors by up to $90 \%$. This involves building an AI-based system that increases communication and transparency between the different actors in the food industry and enables forecasting techniques to better match supply and demand.

The actual ecological benefit of this kind of applications is determined by how companies use the resources that they save, and it is important to avoid rebound effects. If the resources saved are only used to further increase production, this undermines the overall sustainability of the impact. However, if investments are made in more environmental technologies and processes, a positive ecological effect is to be expected. Environmental policy incentives and regulations therefore ought to be designed in a way that encourages companies to increasingly opt for efficiency instead of expansion (UBA 2019). 


\section{Applying Artificial Intelligence and avoiding overkill}

In addition to the examples already mentioned, there are other areas where AI applications could boost sustainable development (see RESET 2020). These show that the potential applications of AI in the area of environmental and climate protection are diverse and that the technology can enable increased energy saving and resource efficiency. From a sustainability perspective, however, it is important not to lose sight of what actually makes sense. This does not only mean assessing whether the resource expenditure of a technology is in proportion to the resource use or resource reduction (c. f. Gährs et al this issue). Instead, we must always begin by asking which solution is the best for each particular problem. This means that in each use case we must consider whether a resource-intensive AI application is really necessary or whether a simple algorithm or a non-technical solution (such as new practices or organisational forms) might also be sufficient (c. f. Colaço this issue). The hype we are currently experiencing with regard to Artificial Intelligence reinforces the tendency to blindly believe in technology - "a new technology can only be better!" - and we run the risk of overkill.

\section{Creating a climate of sustainability}

Both the BMU's Digital Agenda and the White Paper (European Commission 2020) published by the European Commission in February 2020 already highlight the opportunities that AI offers for environmental and climate protection, as well as the regulations needed for sustainable development. But it remains to be seen whether the Digital Agenda, the White Paper and other nascent policy efforts will actually ensure that sustainability plays a more prominent role in AI development.

It is crucial to create sustainable conditions at various levels. Firstly, this includes creating further funding opportunities, as there are currently only a few funding programmes specifically geared towards sustainable AI. Funding programmes such as the "AI Lighthouses for the Environment, Climate, Nature and Resources” from the German Federal Ministry for the Environment (BMU) or programmes by Google and Microsoft are often aimed at individual projects and solutions rather than making AI development more sustainable overall.

Secondly, it is important to create new connections between research communities because there is currently (still) far too little collaboration between research communities working on AI and research communities working on climate and environmental issues. One of the organisations that has set out to build this bridge is Climate Change AI. This alliance of scientists wants to establish a platform for all those who are already working with AI technologies for positive climate impact, and those who would like to, and want to contribute to a reflected and critical discourse on the topic.
In order for AI to be used effectively to shape the complex challenges of our economy, all of the relevant actors from business, civil society, science and politics must work together, especially when it comes to generating, collecting and sharing data (Ellen MacArthur Foundation 2019). We must ensure that data can be shared openly and securely, allowing AI-based applications to be developed in a way that is accountable and involves all actors.

The lack of environmental and climate policy frameworks and incentives also means that sustainability aspects tend to be marginal issues in relation to AI-based technologies in particular, and also with regard to digitalisation in general. However, the more that sustainability is built into our entire digital infrastructure, the more sustainable AI applications will become.

\section{Annotations}

[1] https://powertac.org/

[2] www.carbonintensity.org.uk/

[3] https://reset.org/blog/fuer-mehr-energieeffizienz-bractlet-erschafftdigitale-gebaeude-klone-01062020

[4] https://leanheat.de/

[5] https://reset.org/blog/projekt-reif-kuenstliche-intelligenz-identifiziertfood-waste-entlang-der-lebensmittelkette-030

\section{References}

Blankertz, A. et al. (2020): Themenpapier Datentreuhandmodell. www.stiftung-nv.de/de/publikation/datentreuhandmodelle\#collapsenewsletter_banner_bottom

BMU (2020): Digital Policy Agenda for the Environment. Berlin, Bundesministerium für Umwelt, Naturschutz und nukleare Sicherheit.

Döllner, J. (2020): Geospatial Artificial Intelligence: Potentials of Machine Learning for 3D Point Clouds and Geospatial Digital Twins. In: Journal of Photogrammetry, Remote Sensing and Geoinformation Science 88: 15-24.

Ellen MacArthur Foundation (2019): Artificial Intelligence and the Circular Economy as a Tool to Accelerate the Transition. Cowes, Ellen MacArthur Foundation.

European Commission (2020): White Paper on Artificial Intelligence: a European approach to excellence and trust. Brussels. https://ec.europa.eu/ info/publications/white-paper-artificial-intelligence-european-approachexcellence-and-trust_en

RESET.org (2020): Greenbook (1): Künstliche Intelligenz - Können wir mit Rechenleistung unseren Planeten retten? https://reset.org/blog/ greenbook_01_kuenstliche-intelligenz

UBA (2019): Künstliche Intelligenz im Umweltbereich. Anwendungsbeispiele und Zukunftsperspektiven im Sinne der Nachhaltigkeit. Dessau-Roßlau, Umweltbundesamt.

\section{AUTHOR + CONTACT}

Sarah-Indra Jungblut specializes in the topics of sustainability and digitalization and heads the editorial team of RESET.org.

She also gives lectures and keynotes and moderates talks.

Reset gemeinnützige Stiftungs-GmbH, Neuer Wall 10, 20354 Hamburg. E-Mail: indra.jungblut@reset.org 